\title{
Food and Water Consumption Numeric Result in Standard Unit
}

National Cancer Institute

\section{Source}

National Cancer Institute. Food and Water Consumption Numeric Result in Standard Unit. NCl Thesaurus. Code C119840.

The numerical identifier of a food and water consumption assessment result in standard units. 